\title{
A Five-time-recurring Kawasaki Disease: A Case Report and Literature Review
}

\section{fang chen ( $\nabla$ jhchen_fang@163.com )}

Changsha Central Hospital Affiliated to University of South China: Changsha Central Hospital

\section{Manzhi Wang}

Changsha Central Hospital Affiliated to University of South China: Changsha Central Hospital

\section{Songqing Wei}

Changsha Central Hospital Affiliated to University of South China: Changsha Central Hospital

\section{Research Article}

Keywords: Recurrent, Kawasaki disease, Child, Intravenous immunoglobulin, Case report

Posted Date: January 24th, 2022

DOI: https://doi.org/10.21203/rs.3.rs-1252889/v1

License: (c) (1) This work is licensed under a Creative Commons Attribution 4.0 International License. Read Full License 


\section{Abstract}

\section{Background}

Five-time-recurring Kawasaki Disease without any cardiovascular complications is relatively rare and was first described in 2021.

\section{Case presentation}

We describe a case of five-time-recurring Kawasaki disease (KD) in a 6-year-old boy who had been diagnosed with typical KD without cardiac involvement at age 25 months. Subsequently, the child was hospitalized with KD other 4 times when he was 40, 47, 59, and 66-month-old respetively. In each episode, the boy was characterized by an abrupt intermittently high fever but typical cutaneous and mucosal manifestations were not always presented. Clinical improvement was successfully obtained after intravenous immunoglobulin and (or) aspirin in each time without any cardiovascular damage. Diagnosis was difficult to establish because of the incomplete clinical features and the recurrence of the disease.

\section{Conclusion}

Physicians need to be aware of the differential diagnosis of relapsing KD should be kept in mind while treating a patient with a history of KD. Five-time-recurring Kawasaki Disease is infrequent and will increase the likelihood of poor clinical outcomes if not diagnosed and managed promptly.

\section{Background}

Kawasaki disease (KD) predominantly affects children younger than age 5 and presents with a high fever for at least 5 days combined with rash, oral mucosa changes, bloodshot eyes, extremity abnormalities and cervical lymphadenitis ${ }^{1}$. The diagnosis relies on the compilation of clinical, cardiac ultrasonography and biological data, but there is no specific diagnostic test. The disease can sometimes display incomplete clinical pictures or atypical especially in the recurrent KD that the physician needs to know. Most patients of KD have a uniphasic course, recurrence of KD is rare, and usually occurs within 2 years of the initial episode ${ }^{2}$. Multiple-time-recurring KD is extremely unusual. The recurrence of KD occurs approximately $1.7-3.5 \%$ of all patients diagnosed with $K D$, varying from region to region ${ }^{3,4}$. The recurrent $K D$, especially multiple recurrent case is also rarely reported in China. A PubMed search for "recurrent Kawasaki disease" carried out until November 2021 revealed a total of 685 articles, but very few documented reports of at least 3 relapses of KD. For the first time, we describe the rare case of a 6-yearold boy who presented 5 recurrent episodes KD during 41 months(Table 1), and we also review the literature, focusing on the etiology, diagnosis, and management of the recurrent KD.

\section{Case Presentation}


A boy, full-term born with a neonatal weight of $2.8 \mathrm{~kg}$ and normal neonatal period, presented an abrupt intermittently high fever, when he was 2 years and 1 month old. Fever was combined with unexplained irritability, chapped lips, mucositis, conjunctivitis, rash, stiff edema of both hands and feet and cervical lymphadenopathy on the left. When he was hospitalized, local doctors gave empirical anti-infective treatment for 3 days, but there was no sign of fever abatement. Blood tests showed the following: white blood cell count (WBC) 7,850/ $\mathrm{mm}^{3}$, hemoglobin (Hb) $11.7 \mathrm{~g} / \mathrm{dl}$, C-reactive protein (CRP, n.v. $\otimes 10 \mathrm{mg} / \mathrm{l}$ ) $152 \mathrm{mg} / \mathrm{l}$, erythrosedimentation rate (ESR) $105 \mathrm{~mm} / \mathrm{hr}$. A final diagnosis of KD was established, and treated by intravenous immunoglobulin (IVIG) / aspirin with usual dosages on 4 day. Defervescence was reached after one day at the end of IVIG infusion and typical Kawasaki-like peeling around the extremities(Fig. 1) and anus developed after 10 days, while inflammatory markers were negative. The child was then discharged with that his echocardiographic picture was silent.

15 months later, he was hospitalized again for bellyache and vomiting with increased inflammatory parameters (WBC, 13,740/mm³; CRP, $161.6 \mathrm{mg} /$ l; ESR, 95mm/hr). The next day he developed intermittent high fever which lasted for 7 days. During the course of this episode he only presented cervical lymphadenopathy and transient conjunctivitis. He was treated with penicillin and cephalosporin successively, with poor results. Until the 8th day of the illness the patient was diagnosed as incomplete KD and was treated with aspirin without IVIG. Because his fever had been resolved, and laboratory values had normalized, and echocardiogram was normal. He developed typical Kawasaki-like peeling around the extremities, when he followed up 7 days after discharge.

7 months later, the patient presented again a febrile episode with typical manifestations of KD, accompany with abdominal pain and irritability. Blood tests revealed the following: WBC $19,310 / \mathrm{mm}^{3}, \mathrm{Hb}$ $12.4 \mathrm{~g} / \mathrm{dl}$, CRP $107.5 \mathrm{mg} / \mathrm{l}$, ESR $102 \mathrm{~mm} / \mathrm{hr}$. The child was again hospitalized and treated with IVIG / aspirin following a diagnosis of KD on day 4 of his illness. But fever still persisted at the end of IVIG infusion more than $48 \mathrm{~h}$, so he needed the second dose of IVIG infusion and fever has resolved at the end of the second dose of IVIG infusion. An echocardiography revealed a slight dilation (3.1 mm) (Fig. 2) of the left coronary artery on day 4 , but it soon returned to normal at follow-up without any cardiovascular sequelae.

12 months later, He had intermittent high fever again combined with conjunctivitis, chapped lips, mucositis, and cervical lymph node enlargement. Laboratory tests showed increased inflammatory

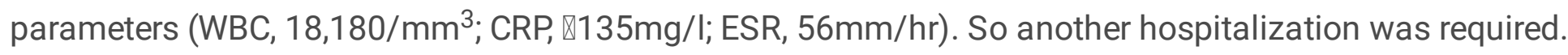
The treatment of antibiotics is ineffective, due to the suspicion of a relapsing KD on day 8 and treated with IVIG/aspirin following a diagnosis of incomplete KD. His cardiological assessment remained normal.

7 months later, when the child was 5.5 years old, He was hospitalized again for persistent fever with increased inflammatory parameters (WBC, 14,540/ $\mathrm{mm}^{3}$; CRP, $\otimes 135 \mathrm{mg} / \mathrm{l} ; \mathrm{ESR}, 60 \mathrm{~mm} / \mathrm{hr}$ ). After 8 days, he was diagnosed incomplete KD fulfilled with 3 of 5 principal symptoms and successfully treated with IVIG / aspirin at the usual dosages. Without any cardiovascular abnormality could be detected on 
echocardiography. Other infectious diseases were excluded in every KD episode. The patient has remained clinically healthy without growth retardation at 29 moths after the last hospitalization.

\section{Discussion And Conclusion}

Kawasaki disease (KD) is an acute, self-limited vasculitis with unknown etiology. Recurrent KD usually occurs within 2 years after the first episode, but multiple recurrent KD is an exceedingly rare occurrence. The literature on recurrent Kawasaki disease was searched in PubMed and limited articles was published. Multiple recurrent KD is only 3 reported cases in literature. Falcini et al ${ }^{5}$ reported a child with recurrence of $K D$ for 4 times in a period of 33 months, each time presented typical KD and were successfully treated without any cardiac sequels. Hamada et $\mathrm{al}^{6}$ described a 1 -year-old male with 4 recurrent episodes of KD within a single year, and his recurrence may be associated with Streptococcus spp, and the third case involved a 10-year-old girl with recurrent KD for 3 episodes, about 4 years apart. In her third episode, because of her older age and atypical presentation led to a delay in diagnosis, she was treated with IVIG until day 13 of illness and was found to have aneurysms of right and left coronary arteries ${ }^{7}$. Our patient, a 5-year-old child presented as much as 5 episodes during 41 months, and his recurrent symptoms were atypical, and was each episode successfully treated with intravenous immunoglobulin and (or) aspirin without any cardiovascular damage.

Factors associated with recurrent KD have been pursued, but the results were not inconsistence. The latest Japan Nationwide Survey ${ }^{8}$ reported that the incidence rate for recurrence was higher among males, patients under the age of 3 years and those who received IVIG or steroid therapy at the initial episode, while cardiovascular complications during the first episode did not affect the recurrence incidence. In a previous retrospective study, Chahal et al showed a lack of association between age at first episode and risk of recurrent $\mathrm{KD}^{9}$. Hirata et al ${ }^{10}$ associated cardiac sequels during the initial episode to be significant risk for recurrent KD. Treatment of IVIG before the fifth day of the disease had been reported to be one of the risk factor for recurrence in children with KD and may be correlated with higher retreatment rate 3,11 . The increased correlation between arterial injury and recurrent KD suggests that more aggressive treatment combines with IVIG may be indicated for the next episode of $\mathrm{KD}^{3}$. During the first and third episode, our patient was treated before the fifth day of illness, which may be one of the reasons for his relapses.

The diagnosis of KD is made according to the criteria of the American Heart Association ${ }^{1}$. The diagnosis of recurrent KD is difficult to establish because of the atypical or incomplete clinical features, and usually requires a combination of cardiac ultrasonography and biological criteria ${ }^{12}$. In our case, he presented complete forms of KD in his first and third episodes, and all of the other 3 relapse episodes were incomplete KD. Incomplete Kawasaki disease we need to be careful to distinguish it from other diseases, such as infectious diseases. In his 3 relapse episodes, our patients who presented 2 or 3 principal symptoms combined with high level biological data (ESR $₫ 40 \mathrm{~mm} / \mathrm{hr}$, CRP $₫ 3.0 \mathrm{mg} / \mathrm{dl}$, platelet count of $\geq 450,000$ after the 7 th day of fever, elevated ALAT level, WBC count of $\geq 15,000 / \mathrm{mm}^{3}$ ), and was invalid 
by anti-infective treatment, were diagnosed as incomplete KD. The multiple recurrent KD also should distinguish from the periodic fever syndrome ${ }^{13}$. The common periodic fever syndrome in children mainly includes Familial Mediterranean Fever (FMF) and High Immunoglobulin-D Syndrome (HIDS). FMF is the most common single-gene mutant recurrent fever syndrome, presenting with episodic fever lasting for about 1-3 days, mostly with a family history ${ }^{14}$. The case in our report was not consistent with FMF. The onset of HIDS occurred in more than $2 / 3$ of the patients within 1 year after birth, with clinical manifestations of periodic fever, growth retardation, and hepatosplenomegaly, and most patients showed elevated IgD and IgA levels ${ }^{15}$. The case in our report was inconsistent with HIDS as well. These diseases are often confusing and need to be carefully identified. It is likely to omit some of these features as the recurrence is often milder and transient than the initial episode, and both the parents and treating doctors may ignore the evolving disease. So physicians need to be aware of the differential diagnosis of relapsing KD should be kept in mind while treating a patient with a history of KD.

High-dose IVIG remains the cornerstone of management, reducing the risk of coronary complications from about $25-4 \%{ }^{16}$, But approximately $10-20 \%$ of patients did not respond to initial high-dose IVIG, which increased the risk of coronary complications to varying degrees ${ }^{11}$. Study has shown that IVIG is more likely to be unresponsive when treating recurrent $\mathrm{KD}^{3}$. However, the strategies for prediction of potential IVIG non-responders and the optimal regime for IVIG-resistant patients remain to be determined ${ }^{17}$. Many experts recommend the second dose IVIG, and retrospective series had suggested safety and efficacy ${ }^{18}$. During the third episode, our patient was still suffering from persistent high fever at least 36 hours following completion of the first dose of IVIG, so he was treated with additional dose IVIG infusion, and he had a slight dilation $(3.1 \mathrm{~mm})$ of the left coronary artery. Fortunately, he returned to normal at the time of follow-up.

To the best of our knowledge, this is a rare case of recurrent KD occurring five times with episodes during 41 months. This case and our literature review should raise awareness that KD can still recur two years after the first episode, often presenting atypically. Recognizing recurrent KD and taking appropriate treatment can result in resolution of symptoms and may decrease the risk of cardiac complications. Hence, the differential diagnosis of relapsing KD should be kept in mind while treating a patient with a history of KD, even if it's more than 2 years since his initial episodes, as aforementioned in the 3 of 4 multiple recurrence patients remaining relapsed after two years of first episodes. Therefore, it is important for every clinician to follow KD patients carefully, even more than 2 years after the first episode.

\section{Abbreviations}

CRP, C-reactive protein; ASA, aspirin; ALAT, alanine aminotransferase; KLS, Kawasaki-like syndrome; ASAT, aspartate aminotransferase; ESR, erythrocyte sedimentation rate; IVIG, iv human gamma globulin; TR, tricuspid regurgitation; SD LCA, Slight dilation left coronary artery; +, present; - , absent.

\section{Declarations}




\section{Ethics approval and consent to participate}

Not applicable.

\section{Consent to publish}

Both written and verbal consent to publish the case was obtained from patients' parents.

\section{Availability of data and materials}

Not applicable.

\section{Competing interests}

The authors declare no conflict of interest.

\section{Funding}

Not applicable.

\section{Authors' contributions}

FC: Wrote case presentation and literature review.

MW: Diagnosed the case.

SW: Reviewed the manuscript.

All authors have read and approved the final version of the manuscript.

\section{Acknowledgements}

Not applicable.

\section{References}

1. Sosa T, Brower L, Divanovic A. Diagnosis and Management of Kawasaki Disease. JAMA Pediatr. 2019;173(3):278-9.

2. Guleria S, Pilania RK, Jindal AK, et al. Recurrent Kawasaki disease at a tertiary care center in Chandigarh, North West India: 24 years of clinical experience. Int J Rheum Dis. 2019;22(7):1183-7.

3. Maddox RA, Holman RC, Uehara R, et al. Recurrent Kawasaki disease: USA and Japan. Pediatr Int. 2015;57(6):1116-20. 
4. Makino N, Nakamura Y, Yashiro M, et al. Descriptive epidemiology of Kawasaki disease in Japan, 2011-2012: from the results of the 22nd nationwide survey. J Epidemiol. 2015;25(3):239-45.

5. Falcini F, D'Adamo G, Rigante D. A four-time-recurring typical complete Kawasaki syndrome successfully treated with intravenous immunoglobulin: a case report with literature review. Rheumatol Int. 2013;33(10):2653-5.

6. Hamada H, Sekizuka T, Oba K, et al. Comprehensive pathogen detection associated with four recurrent episodes of Kawasaki diseasein a patient during a single year using next-generation sequencing. JMM Case Rep. 2016;3(1):e005019.

7. Goswami N, Marzan K, De Oliveira E, et al. Recurrent Kawasaki Disease: A Case Report of Three Separate Episodes at >4-Year Intervals. Children (Basel). 2018;5(11). pii: E155.

8. Sudo D, Nakamura Y. Nationwide surveys show that the incidence of recurrent Kawasaki disease in Japan has hardly changed over the last 30 years. Acta Paediatr. 2017;106(5):796-800.

9. Luo G, Pan SL, Wang SB, et al. Clinical features of children with recurrent Kawasaki disease: a Meta analysis. Zhongguo Dang Dai Er Ke Za Zhi. 2020;22(12):1306-12.

10. Hirata $S$, Nakamura $Y$, Yanagawa $H$. Incidence rate of recurrent Kawasaki disease and related risk factors: from the results of nationwide surveys of Kawasaki disease in Japan. Acta Paediatr. 2001;90(1):40-4.

11. Maddox RA, Holman RC, Uehara R, et al. Recurrent Kawasaki disease: USA and Japan. Pediatr Int. 2015;57(6):1116-20.

12. Hongkun J, Zhiliang Y. Severe Recurrent Fever Episodes With Clinical Diagnosis of Hemophagocytic Lymphohistiocytosis, Incomplete Kawasaki Disease and Systemic-Onset Juvenile Idiopathic Arthritis: A Case Report and Literature Review. Front Pediatr. 2020;8:93.

13. Sag E, Bilginer Y, Ozen S. Autoinflammatory Diseases with Periodic Fevers. Curr Rheumatol Rep. 2017;19(7):41.

14. Van Gorp H, Huang L, Saavedra P, et al. Blood-based test for diagnosis and functional subtyping of familial Mediterranean fever. Ann Rheum Dis. 2020;79(7):960-8.

15. Peter McNaughton S, Willcocks SH, Lum, et al. Making a diagnosis of periodic fever syndrome: Experience from a single tertiary centre. J Paediatr Child Health. 2021. doi:10.1111/jpc.15722. Online ahead of print.

16. McCrindle BW, Rowley AH. Improving coronary artery outcomes for children with Kawasaki disease. Lancet. 2019;393(10176):1077-8.

17. Kibata T, Suzuki Y, Hasegawa S, et al. Coronary artery lesions and the increasing incidence of Kawasaki disease resistant to initial immunoglobulin. Int J Cardiol. 2016;1(214):209-15.

18. Song MS. Predictors and management of intravenous immunoglobulin-resistant Kawasaki disease. Korean J Pediatr. 2019;62(4):119-23.

\section{Tables}




\section{Table 1}

Characteristic and examination results of the patient 


\begin{tabular}{|c|c|c|c|c|c|}
\hline & episodes & & & & \\
\hline Characteristic or variable & first & second & third & fourth & fifth \\
\hline Age, $m$ & 25 & 40 & 47 & 59 & 66 \\
\hline Date of onset & 2016.01 .28 & 2017.04 .22 & 2017.11.22 & 2018.11.18 & 2019.06.07 \\
\hline $\begin{array}{l}\text { Time since previous } \\
\text { case }\end{array}$ & - & $15 \mathrm{mo}$ & $7 \mathrm{mo}$ & $12 \mathrm{mo}$ & $7 \mathrm{mo}$ \\
\hline Treatment & IVIG/ASA & ASA & IVIG/ASA & IVIG/ASA & IVIG/ASA \\
\hline \multicolumn{6}{|l|}{ Symptoms of KLS } \\
\hline Fever & + & + & + & + & + \\
\hline Rash & + & - & - & - & - \\
\hline Desquamation & + & + & + & - & - \\
\hline Extremity changes & + & - & + & - & - \\
\hline Conjunctivitis & + & + & + & + & + \\
\hline Oropharynx changes & + & - & + & + & - \\
\hline $\begin{array}{l}\text { cervical } \\
\text { lymphadenopathy, } ₫ 15 \mathrm{~mm}\end{array}$ & + & + & + & + & + \\
\hline Abdominal pain & + & + & + & - & - \\
\hline Nausea & + & + & - & - & - \\
\hline Diarrhea & - & - & - & - & - \\
\hline \multicolumn{6}{|l|}{ Results of laboratory studies } \\
\hline WBC count, cells $\times 10^{3} / \mathrm{mm}^{3}$ & 7,850 & 13,740 & 19,310 & 18,180 & 14,540 \\
\hline Platelet count, cells $\times 10^{3} / \mu \mathrm{L}$ & 261 & 277 & 167 & 408 & 304 \\
\hline CRP level, mg/dL & 152 & 161.6 & 107.5 & \135 & $\mathbb{1 1 3 5}$ \\
\hline $\mathrm{ESR}, \mathrm{mm} / \mathrm{hr}$ & 105 & 95 & 102 & 56 & 60 \\
\hline ASAT level, IU/L & 34.1 & 151 & 18.5 & 150 & 27 \\
\hline ALAT level, IU/L & 154.2 & 275 & 54.7 & 214 & 16 \\
\hline \multicolumn{6}{|l|}{ Results of other studies } \\
\hline Echocardiogram & Normal & Trace TR & SD LCA & Normal & Trace TR \\
\hline Chest X-ray & - & - & Pneumonia & - & Bronchitis \\
\hline
\end{tabular}


Figures

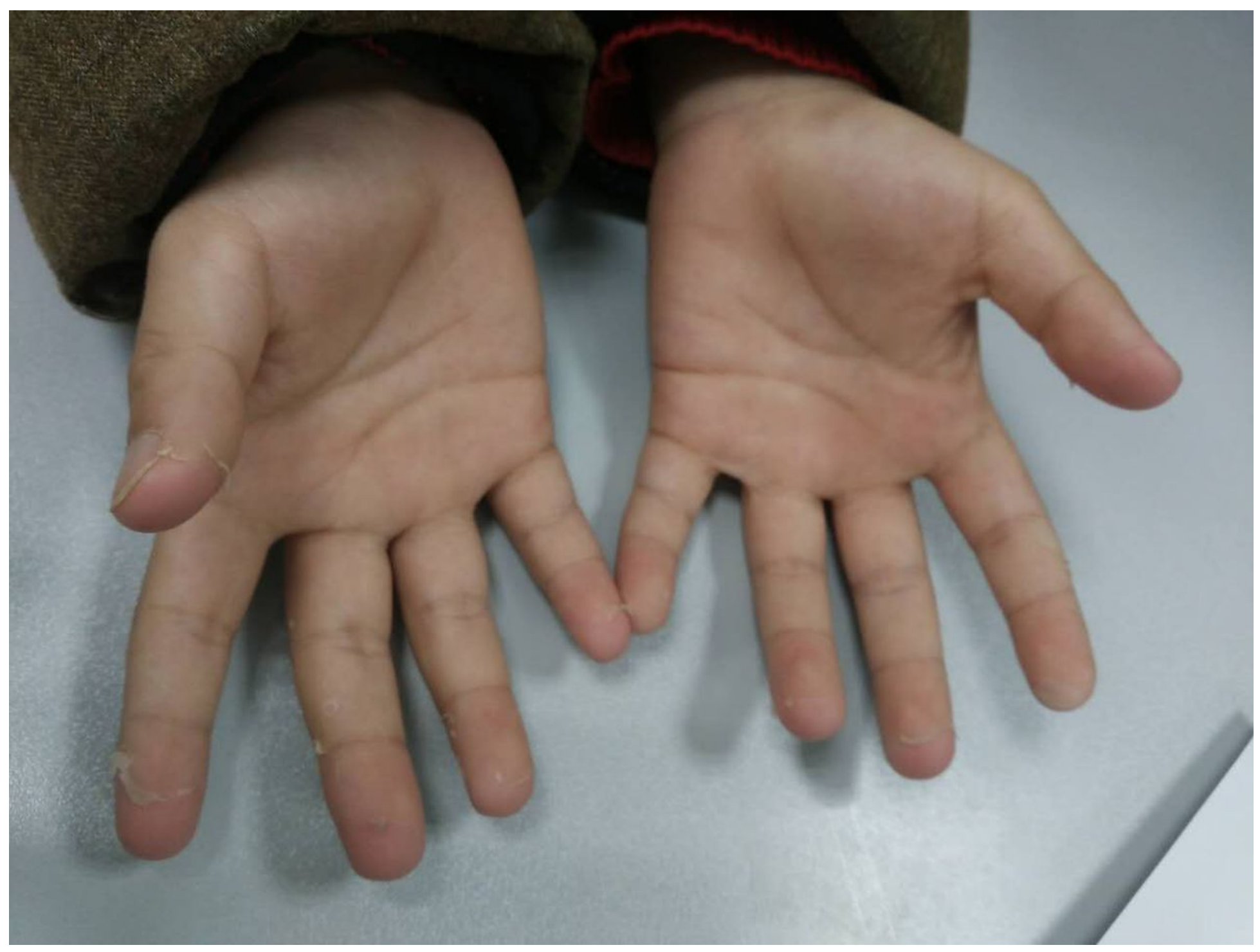

Figure 1

Photograph showing desquamation of palms 


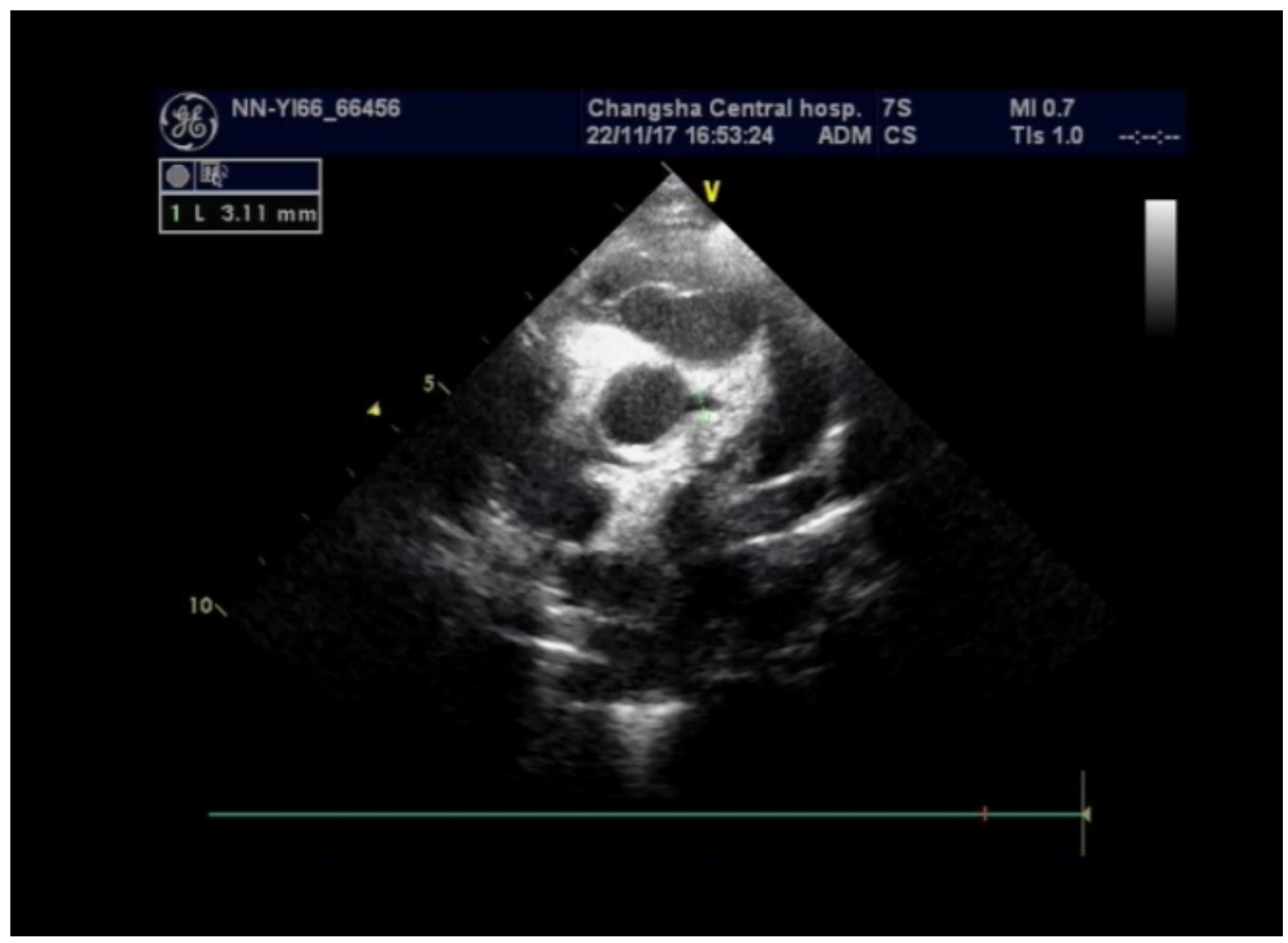

Figure 2

Photograph showing slight dilation of left coronary artery 